\title{
ASYMPTOTIC FORMULÆ FOR THE NUMBER OF PARTITIONS OF A MULTI-PARTITE NUMBER
}

\author{
by M. M. ROBERTSON \\ (Received 20th January 1960)
}

\section{Introduction}

A multi-partite number of order $j$ is a $j$ dimensional vector, the components of which are non-negative rational integers. A partition of $\left(n_{1}, n_{2}, \ldots, n_{j}\right)$ is a solution of the vector equation

$$
\sum_{k}\left(n_{1 k}, n_{2 k}, \ldots, n_{j k}\right)=\left(n_{1}, n_{2}, \ldots, n_{j}\right)
$$

in multi-partite numbers other than $(0,0, \ldots, 0)$. Two partitions, which differ only in the order of the multi-partite numbers on the left-hand side of (1), are regarded as identical. We denote by $p_{1}\left(n_{1}, \ldots, n_{j}\right)$ the number of different partitions of $\left(n_{1}, \ldots, n_{j}\right)$ and by $p_{2}\left(n_{1}, \ldots, n_{j}\right)$ the number of those partitions in which no part has a zero component. Also, we write $p_{3}\left(n_{1}, \ldots, n_{j}\right)$ for the number of partitions of $\left(n_{1}, \ldots, n_{j}\right)$ into different parts and $p_{4}\left(n_{1}, \ldots, n_{j}\right)$ for the number of partitions into different parts none of which has a zero component.

By adaptations to $j>1$ of the celebrated Hardy-Ramanujan method (1) for the $j=1$ case, several authors have recently obtained asymptotic expressions for $p_{r}\left(n_{1}, \ldots, n_{j}\right)$, which are valid under certain restrictions upon the relative rates at which the different $n_{l}$ tend to infinity. Auluck (3) obtained a formula for $p_{1}\left(n_{1}, n_{2}\right)$, where $n_{1}$ and $n_{2}$ are large but of the same order of magnitude, i.e. the ratio $n_{1} / n_{2}$ is bounded above and below, and, under the same conditions, Wright (7) found asymptotic expressions for $p_{r}\left(n_{1}, n_{2}\right)$, where $r=1,2,3$ and 4. In his article, Wright also gave without proof the first few terms of an asymptotic formula for $\log p_{2}\left(n_{1}, \ldots, n_{j}\right)$, where every $n_{l}$ is of the same order of magnitude. Meinardus (4) had just previously published a paper in which he had found the first term of this formula for multi-partites. Later, Wright (8) obtained asymptotic expressions for $p_{r}\left(n_{1}, n_{2}\right)$ which hold for $n_{1}^{\frac{1}{2}+\varepsilon_{1}}<n_{2}<n_{1}^{2-\varepsilon_{2}}$, where $r=1,2,3$ and 4 and $\varepsilon_{1}$ and $\varepsilon_{2}$ are any fixed positive numbers. This is a substantial relaxation of the restrictions imposed upon $n_{1}$ and $n_{2}$ in both (3) and (7). In his article, Auluck also obtained a formula for $p_{1}\left(n_{1}, n_{2}\right)$ when $n_{2}$ is fixed and $n_{1}$ is large, and Nanda (5) has shown that this formula remains valid when $n_{2}$ is large, provided that $n_{2}=o\left(n_{1}^{\frac{1}{4}}\right)$. In an article in preparation, I extend Wright's method to derive formulæ for $p_{r}\left(n_{1}, \ldots, n_{j}\right)$ for $r=1,2,3$ and 4 and $n_{1} \ldots n_{j}<\bar{n}^{j+1-\varepsilon_{3}}$, where $\bar{n}=\min n_{l}$ and $\varepsilon_{3}$ is any fixed positive number. In this article, I evaluate $p_{r}\left(n_{1}, \ldots, n_{j}\right)$ for $r=1$ and 3 when one particular $n_{l}$ tends to infinity more rapidly than the fourth power of every other $n_{l}$ by means of an extension of Nanda's method $\dagger$ and I also

$\dagger$ This problem was suggested to me by Professor E. M. Wright to whom I am also grateful for much valuable advice in the course of the investigation. 
obtain an asymptotic formula for $p_{r}\left(n_{1}, \ldots, n_{j}\right)$ for $r=2$ and 4 when one particular $n_{l}$ tends to infinity more slowly than the cube root of every other $n_{l}$.

The letters $h, k, l, m, n, N, q, r, R, R^{\prime}$ and $v$ represent non-negative integers which may be fixed or variable according to the context and $j$ is used for a fixed integer greater than unity. $C$ is a positive number, not necessarily the same at each occurrence, which may depend upon $j$ but not upon any $n_{l}$. When there is no statement to the contrary, the symbols $O(), o()$ and $\sim$ refer to the passage of the $n_{l}$ to infinity.

\section{Asymptotic Formulæ for $p_{r}\left(n_{1}, \ldots, n_{j}\right)$}

It is easily seen that $p_{r}\left(n_{1}, \ldots, n_{j}\right)$ is a symmetric function of $n_{1}, \ldots, n_{j}$ and so, without any loss of generality, we may suppose that $n_{1} \geqq n_{2} \geqq \ldots \geqq n_{j}$. Nanda (5) has shown that the asymptotic formula

$$
p_{1}\left(n_{1}, n_{2}\right) \sim\left(\frac{6 n_{1}}{\pi^{2}}\right)^{\frac{1}{2} n_{2}}\left\{4 \sqrt{ } 3 n_{1}\left(n_{2} !\right)\right\}^{-1} \exp \left\{\pi /\left(\frac{2 n_{1}}{3}\right)\right\}
$$

as $n_{1} \rightarrow \infty$ holds for $n_{2}=o\left(n_{1}^{\frac{1}{1}}\right)$. If we write $R_{j}=\sum_{l=2}^{j} n_{l}$, the above formula is seen to be a particular case of the following more general theorem.

Theorem 1. If $n_{l}=o\left(n_{1}^{\frac{1}{1}}\right)$ for $2 \leqq l \leqq j$, then

$$
p_{1}\left(n_{1}, \ldots, n_{j}\right) \sim\left(\frac{6 n_{1}}{\pi^{2}}\right)^{\frac{1}{2} R_{j}}\left(4 \sqrt{3} 3 n_{1} \prod_{l=2}^{j} n_{l} !\right)^{-1} \exp \left\{\pi /\left(\frac{2 n_{1}}{3}\right)\right\}
$$

as $n_{1} \rightarrow \infty$.

Asymptotic formulæ can also be obtained for $p_{r}\left(n_{1}, \ldots, n_{j}\right)$ when $r=2,3$ and 4 , and indeed the following theorems will be proved.

Theorem 2. If $n_{j}=o\left(n_{l}^{\frac{1}{1}}\right)$ for $1 \leqq l \leqq j-1$, then

$$
p_{2}\left(n_{1}, \ldots, n_{j}\right) \sim\left(n_{1} \ldots n_{j-1}\right)^{n_{j}-1}\left\{\left(n_{j}-1\right) !\right\}^{1-j}\left(n_{j} !\right)^{-1}
$$

as $n_{l} \rightarrow \infty$ for $1 \leqq l \leqq j-1$.

Theorem 3. If $n_{l}=o\left(n_{1}^{4}\right)$ for $2 \leqq l \leqq j$, then

as $n_{1} \rightarrow \infty$.

$$
p_{3}\left(n_{1}, \ldots, n_{j}\right) \sim\left(\frac{12 n_{1}}{\pi^{2}}\right)^{\frac{1}{R_{j}}}\left(4.3^{\frac{1}{2}} n_{1}^{\frac{3}{2}} \prod_{l=2}^{j} n_{l} !\right)^{-1} \exp \left\{\pi /\left(\frac{n_{1}}{3}\right)\right\}
$$

Theorem 4. If $n_{j}=o\left(n_{l}^{\frac{7}{1}}\right)$ for $1 \leqq l \leqq j-1$, then

$$
p_{4}\left(n_{1}, \ldots, n_{j}\right) \sim\left(n_{1} \ldots n_{j-1}\right)^{n_{j}-1}\left\{\left(n_{j}-1\right) !\right\}^{1-j}\left(n_{j} !\right)^{-1}
$$

as $n_{l} \rightarrow \infty$ for $1 \leqq l \leqq j-1$.

\section{Two Lemmas}

We put

$$
\alpha_{1}\left(h_{1}, \ldots, h_{j}\right)=\alpha_{2}\left(h_{1}, \ldots, h_{j}\right)=\left(1-x_{1}^{h_{1}} \ldots x_{j}^{h_{j}}\right)^{-1}
$$


and

$$
\alpha_{3}\left(h_{1}, \ldots, h_{j}\right)=\alpha_{4}\left(h_{1}, \ldots, h_{j}\right)=1+x_{1}^{h_{1}} \ldots x_{j}^{h_{j}},
$$

where $\left|x_{1}\right|<1$ for $1 \leqq l \leqq j$. Then we write

$$
f_{r}\left(x_{1}, \ldots, x_{j}\right)=\prod_{h_{1}, \ldots, h_{j}} \alpha_{r}\left(h_{1}, \ldots, h_{j}\right),
$$

where, for $r=2$ and $4, h_{1}, \ldots, h_{j}$ each take all positive integral values, while, for $r=1$ and $3, h_{1}, \ldots, h_{j}$ each take all non-negative integral values except $h_{1}=\ldots=h_{j}=0$. If we put $p_{r}(0,0, \ldots, 0)=1$, we can easily verify from the definitions of $p_{r}\left(n_{1}, \ldots, n_{j}\right)$ that

$$
f_{r}\left(x_{1}, \ldots, x_{j}\right)=\sum_{n_{1}=0}^{\infty} \ldots \sum_{n_{j}=0}^{\infty} p_{r}\left(n_{1}, \ldots, n_{j}\right) x_{1}^{n_{1}} \ldots x_{j}^{n_{j}}
$$

for $r=1,2,3$ and 4 .

Before proceeding with the proof of Theorem 1, we require the following lemma.

Lemma 1. If, when $2 \leqq k<C$ and $n_{l}=o\left(n_{1}^{\frac{1}{1}}\right)$ for $2 \leqq l \leqq k$,

$p_{1}\left(n_{1}, \ldots, n_{k}\right)=\left(\frac{6 n_{1}}{\pi^{2}}\right)^{\frac{1}{2} R_{k}}\left(4 \sqrt{3} n_{1} \prod_{l=2}^{k} n_{l} !\right)^{-1} \exp \left\{\pi /\left(\frac{2 n_{1}}{3}\right)\right\}\left\{1+\sum_{r=1}^{R_{k}+1} O\left(r n_{1}^{-\frac{1}{2}}\right)\right\}$

as $n_{1} \rightarrow \infty$, and if

$$
f_{1}\left(x_{1}, \ldots, x_{k}\right) \prod_{n=1}^{q} \prod_{l=1}^{k}\left(1-x_{l}^{N_{h}}\right)^{-1}=\sum_{n_{1}=0}^{\infty} \ldots \sum_{n_{k}=0}^{\infty} \bar{\omega}_{N_{1} \ldots N_{g}}\left(n_{1}, \ldots, n_{k}\right) x_{1}^{n_{1}} \ldots x_{k}^{n_{k}},
$$

where $N_{h} \geqq 1$ for $1 \leqq h \leqq q$, then, provided that

$$
R^{\prime}=\sum_{h=1}^{q} N_{h}=o\left(n_{1}^{\frac{1}{1}}\right)
$$

$\bar{\omega}_{N_{1} \ldots N_{q}}\left(n_{1}, \ldots, n_{k}\right)$

$=\left(\frac{6 n_{1}}{\pi^{2}}\right)^{\frac{1}{2}\left(R_{k}+q\right)}\left(4 \sqrt{ } 3 n_{1} \prod_{h=1}^{q} N_{h} \prod_{l=2}^{k} n_{l} !\right)^{-1} \exp \left\{\pi /\left(\frac{2 n}{3}\right)\right\}\left\{1+\sum_{r=1}^{R_{k}+R^{\prime}+1} O\left(r n_{1}^{-\frac{1}{2}}\right)\right\} \ldots$

as $n_{1} \rightarrow \infty$, where the constants implicit in the " $O$ " terms on the right-hand side of (2) are independent of $q$.

Before we prove Lemma 1, we prove

Lemma 2. If $k \geqq 0, m \geqq 1$ and $\rho$ is any fixed positive number, then

$$
\begin{aligned}
\sum_{r=0}^{\left[\frac{1}{2} m^{-1} n\right]}(n-m r)^{\frac{1}{2}(k-3)} \exp \{\rho \sqrt{ }(n-m r)\} \\
=(2 / \rho m) n^{\frac{1}{2}(k-2)} \exp (\rho \sqrt{ } n)\left\{1+O\left(k n^{-\frac{1}{2}}\right)+O\left(m n^{-\frac{1}{2}}\right)\right\}
\end{aligned}
$$

as $n \rightarrow \infty$, provided that $k=o\left(n^{\frac{1}{2}}\right)$ and $m=o\left(n^{\frac{1}{2}}\right)$.

In order to prove Lemma 1, it is sufficient to show that Lemma 2 holds when $k$ and $m$ are each $o\left(n^{\frac{1}{4}}\right)$, but it is evident from the following proof that Lemma 2 remains true provided that $k$ and $m$ are each $o\left(n^{\frac{1}{2}}\right)$. If $0 \leqq t \leqq \frac{1}{2}$

E.M.S. - C 
34

M. M. ROBERTSON

and $k \geqq 2$, then

$$
1-\frac{1}{2} k t \leqq(1-t)^{\frac{1}{2}} \cdot \leqq 1
$$

Also, for $0 \leqq t \leqq \frac{1}{2}$,

and

$$
\begin{gathered}
1-\frac{1}{2} t-\frac{1}{2} t^{2} \leqq(1-t)^{\frac{1}{2}} \leqq 1-\frac{1}{2} t \\
1 \leqq(1-t)^{-\frac{1}{2}} \leqq(1-t)^{-1} \leqq(1-t)^{-\frac{3}{2}} \leqq 1+4 t
\end{gathered}
$$

and, for all $t \geqq 0$,

$1-t \leqq \exp (-t)$

Hence, for all $k \geqq 0$,

$$
\begin{aligned}
\sum_{r=0}^{\left[\frac{1}{2} m^{-1} n\right]}\left(1-\frac{k m r}{2 n}\right)\left(1-\frac{\rho m^{2} r^{2}}{2 n^{\frac{3}{2}}}\right) \exp \left(-\frac{\rho m r}{2 \sqrt{ } n}\right) & \leqq \Sigma^{*} n^{-\frac{1}{2}(k-3)} \exp (-\rho \sqrt{ } n) \\
& \leqq \sum_{r=0}^{\left[\frac{1}{2} m^{-1} n\right]}\left(1+\frac{4 m r}{n}\right) \exp \left(-\frac{\rho m r}{2 \sqrt{ } n}\right),
\end{aligned}
$$

where $\Sigma^{*}$ denotes the sum on the left-hand side of (3). Therefore, since $k=o\left(n^{\frac{1}{2}}\right)$ and $m=o\left(n^{\frac{1}{2}}\right)$,

$$
\begin{aligned}
&(2 \sqrt{ } n / \rho m)\left\{1+O\left(k n^{-\frac{1}{2}}\right)+O\left(m n^{-\frac{1}{2}}\right)\right\} \\
& \leqq \Sigma^{*} n^{-\frac{1}{2}(k-3)} \exp (-\rho \sqrt{ } n) \leqq(2 \sqrt{ } n / \rho m)\left\{1+O\left(m n^{-\frac{1}{2}}\right)\right\}
\end{aligned}
$$

and Lemma 2 follows immediately.

We now prove Lemma 1. From the definition of $\bar{\omega}_{N_{1}}\left(n_{1}, \ldots, n_{k}\right)$, we have

$$
\bar{\omega}_{N_{1}}\left(n_{1}, \ldots, n_{k}\right)=\sum_{v_{l}=0}^{\left[N_{1}^{-1} n_{1}\right]} \ldots \sum_{v_{k}=0}^{\left[N_{1}^{-1} n_{k}\right]} p_{1}\left(n_{1}-N_{1} v_{1}, \ldots, n_{k}-N_{1} v_{k}\right) .
$$

From Lemma 2, we obtain

$$
\begin{aligned}
& \sum_{v_{1}=0}^{\left[\frac{1}{2} N_{1}^{-1} n_{1}\right]} p_{1}\left(n_{1}-N_{1} v_{1}, n_{2}, \ldots, n_{k}\right)=\left(\frac{6 n_{1}}{\pi^{2}}\right)^{\frac{1}{2}\left(R_{k}+1\right)}\left(4 \sqrt{3} n_{1} N_{1} \prod_{l=2}^{k} n_{l} !\right)^{-1} \\
& \quad \times \exp \left\{\pi /\left(\frac{2 n_{1}}{3}\right)\right\}\left\{1+\sum_{r=1}^{R_{k}+1} O\left(r n_{1}^{-\frac{1}{2}}\right)+O\left(\left\{R_{k}+3\right\} n_{1}^{-\frac{1}{2}}\right)+O\left(N_{1} n_{1}^{-\frac{1}{2}}\right)\right\} \\
& =\left(\frac{6 n_{1}}{\pi^{2}}\right)^{\frac{1}{2}\left(R_{k}+1\right)}\left(4 \sqrt{3} n_{1} N_{1} \prod_{l=2}^{k} n_{l} !\right)^{-1} \exp \left\{\pi /\left(\frac{2 n_{1}}{3}\right)\right\}\left\{1+\sum_{r=1}^{R_{k}+N_{1}+1} O\left(r n_{1}^{-\frac{1}{2}}\right)\right\}
\end{aligned}
$$

Clearly, when $v_{1}>\left[\frac{1}{2} N_{1}^{-1} n_{1}\right]$,

$$
p_{1}\left(n_{1}-N_{1} v_{1}, n_{2}, \ldots, n_{k}\right) \leqq p_{1}\left(\left[\frac{1}{2} n_{1}\right], n_{2}, \ldots, n_{k}\right)
$$

and therefore,

$$
\begin{aligned}
\sum_{v_{1}=\left[\frac{1}{2} N_{1}^{-1} n_{1}\right]+1}^{\left[N_{1}^{-1} n_{1}\right]} p_{1}\left(n_{1}-\right. & \left.N_{1} v_{1}, n_{2}, \ldots, n_{k}\right) \\
& <C N_{1}^{-1} n_{1}\left(\frac{3 n_{1}}{\pi^{2}}\right)^{\frac{1}{2} R_{k}}\left(4 \sqrt{ } 3 n_{1} \prod_{l=2}^{k} n_{l} !\right)^{-1} \exp \left\{\pi /\left(\frac{n_{1}}{3}\right)\right\} .
\end{aligned}
$$

https://doi.org/10.1017/S0013091500025025 Published online by Cambridge University Press 
It follows that

$$
\begin{aligned}
\sum_{v_{1}=0}^{\left[N_{1}^{-1} n_{1}\right]} p_{1}\left(n_{1}-N_{1} v_{1}, n_{2}, \ldots, n_{k}\right) & \left(\frac{6 n_{1}}{\pi^{2}}\right)^{\frac{1}{2}\left(R_{k}+1\right)}\left(4 \sqrt{3} n_{1} N_{1} \prod_{l=2}^{k} n_{l} !\right)^{-1} \exp \left\{\pi /\left(\frac{2 n_{1}}{3}\right)\right\} \\
& \times\left\{1+\sum_{r=1}^{R_{k}+N_{1}+1} O\left(r n_{1}^{-\frac{1}{2}}\right)\right\} .
\end{aligned}
$$

Hence,

$$
\begin{aligned}
& \bar{\omega}_{N_{1}}\left(n_{1}, \ldots, n_{k}\right) \\
& =\left(\frac{6 n_{1}}{\pi^{2}}\right)^{\frac{1}{2}\left(R_{k}+1\right)} \cdot\left(4 \sqrt{3} n_{1} N_{1} \prod_{l=2}^{k} n_{l} !\right)^{-1} \exp \left\{\pi /\left(\frac{2 n_{1}}{3}\right)\right\}\left\{1+\sum_{r=1}^{R_{k}+N_{1}+1} O\left(r n_{1}^{-\frac{1}{2}}\right)\right\} \\
& \quad \times \prod_{l=2}^{k}\left\{1+\frac{n_{l} !}{\left(n_{l}-N_{1}\right) !}\left(\frac{6 n_{1}}{\pi^{2}}\right)^{-\frac{1}{2} N_{1}}+\frac{n_{l} !}{\left(n_{l}-2 N_{1}\right) !}\left(\frac{6 n_{1}}{\pi^{2}}\right)^{-N_{1}}\right. \\
& \left.+\ldots+\frac{n_{l} !}{\left(n_{l}-\left[N_{1}^{-1} n_{l}\right] N_{1}\right) !}\left(\frac{6 n_{1}}{\pi^{2}}\right)^{-\frac{1}{2}\left[N_{1}^{-1} n_{l}\right] N_{1}}\right\} \\
& =\left(\frac{6 n_{1}}{\pi^{2}}\right)^{\frac{1}{2}\left(R_{k}+1\right)}\left(4 \sqrt{3} n_{1} N_{1} \prod_{l=2}^{k} n_{l} !\right)^{-1} \exp \left\{\pi /\left(\frac{2 n_{1}}{3}\right)\right\}\left\{1+\sum_{r=1}^{R_{k}+N_{1}+1} O\left(r n_{1}^{-\frac{1}{2}}\right)\right\},
\end{aligned}
$$

since $n_{l}=o\left(n_{1}^{\frac{1}{t}}\right)$ for $2 \leqq l \leqq k$. Next, if we assume that (2) holds for any positive integers $q, N_{1}, \ldots, N_{q}$ such that $\sum_{h=1}^{q} N_{h}=o\left(n_{1}^{\frac{1}{t}}\right)$, an argument exactly similar to the above shows that (2) remains true when $q$ is replaced by $q+1$ and $N_{q+1}=o\left(n_{1}^{\frac{1}{4}}\right)$. Lemma 1 follows immediately by inductive reasoning.

\section{Proof of Theorem 1}

The generating function of $p_{1}\left(n_{1}, \ldots, n_{k+1}\right)$ is

$$
f_{1}\left(x_{1}, \ldots, x_{k+1}\right)=\prod_{h_{1}}, \ldots, h_{k+1}\left(1-x_{1}^{h_{1}} \ldots x_{k+1}^{h_{k}+1}\right)^{-1},
$$

where the product is taken over all non-negative integers $h_{1}, \ldots, h_{k+1}$ except $h_{1}=\ldots=h_{k+1}=0$. It follows that, for $k \geqq 1$,

$$
f_{1}\left(x_{1}, \ldots, x_{k+1}\right)=f_{1}\left(x_{1}, \ldots, x_{k}\right) \prod_{h_{1}, \ldots, h_{k+1}}^{\prime}\left(1-x_{1}^{h_{1}} \ldots x_{k+1}^{h_{k+1}}\right)^{-1},
$$

where the latter product is taken over all non-negative $h_{1}, \ldots, h_{k}$ and all positive $h_{k+1}$. We write

$$
\prod_{h_{1}, \ldots, h_{k+1}}^{\prime}\left(1-x_{1}^{h_{1}} \ldots x_{k+1}^{h_{k+1}}\right)^{-1}=1+\sum_{n=1}^{\infty} A_{n} x_{k+1}^{n}
$$

where

$$
A_{n}=\sum_{(n)} \prod_{m} c_{v_{m}}
$$


the sum being taken over all partitions of $n$ of the form $n=\sum_{m} m v_{m}$ and the product over all the different parts $m$ of the partition, and $c_{n}$ is the coefficient of $y^{n}$ in $g(y)$, where

$$
g(y)=\prod_{h_{1}=0}^{\infty} \ldots \prod_{h_{k}=0}^{\infty}\left(1-x_{1}^{h_{1}} \ldots x_{k}^{h_{k}} y\right)^{-1}
$$

and $|y|<1$. Also

$$
\begin{aligned}
\log g(y) & =-\sum_{h_{1}=0}^{\infty} \ldots \sum_{h_{k}=0}^{\infty} \log \left(1-x_{1}^{h_{1}} \ldots x_{k}^{h_{k}} y\right) \\
& =\sum_{h_{1}=0}^{\infty} \ldots \sum_{h_{k}=0}^{\infty} \sum_{r=1}^{\infty} r^{-1} x_{1}^{r h_{1}} \ldots x_{k}^{r h_{k}} y^{r} \\
& =\sum_{r=1}^{\infty} r^{-1} y^{r} \prod_{l=1}^{k}\left(1-x_{l}^{r}\right)^{-1}
\end{aligned}
$$

and so,

$$
g(y)=\exp \left\{\sum_{r=1}^{\infty} r^{-1} y^{r} \prod_{l=1}^{k}\left(1-x_{l}^{r}\right)^{-1}\right\}
$$

It follows that

$$
c_{n}=\sum_{(n)} \prod_{m}\left(v_{m}^{\prime} !\right)^{-1}\left\{m \prod_{l=1}^{k}\left(1-x_{l}^{m}\right)\right\}^{-v_{m}^{\prime}}
$$

where the sum is taken over all partitions of $n$ of the form $n=\sum_{m} m v_{m}^{\prime}$ and the product over all the different parts $m$ of the partition.

We now prove by induction that, if $n_{l}=o\left(n_{1}^{\frac{b}{b}}\right)$ for $2 \leqq l \leqq j$, then

$$
\begin{aligned}
& p_{1}\left(n_{1}, \ldots, n_{j}\right) \\
& \quad=\left(\frac{6 n_{1}}{\pi^{2}}\right)^{\frac{1}{2} R_{j}}\left(4 \sqrt{3} 3 n_{1} \prod_{l=2}^{j} n_{l} !\right)^{-1} \exp \left\{\pi /\left(\frac{2 n_{1}}{3}\right)\right\}\left\{1+\sum_{r=1}^{R_{j}+1} O\left(r n_{1}^{-2}\right)\right\}
\end{aligned}
$$

as $n_{1} \rightarrow \infty$. In (5), Nanda has already demonstrated that (8) is true for $j=2$. Here, we assume that (8) holds for $j=k$, where $k$ is any fixed positive integer greater than unity. From (4) and (5), $p_{1}\left(n_{1}, \ldots, n_{k+1}\right)$ is equal to the coefficient of $x_{1}^{n_{1}} \ldots x_{k}^{n_{k}}$ in $A_{n_{k+1}} f_{1}\left(x_{1}, \ldots, x_{k}\right)$. We see from (6) that there is a one-to-one correspondence between the terms of $A_{n}$ and the partitions of $n$. We therefore divide the partitions of $n$ into classes in which each partition has the same number of parts and we make a corresponding division of the terms of $A_{n}$ into sets. For $0 \leqq q \leqq n-1$, the $(q+1)$ th set has $p_{1}^{(n-q)}(n)$ terms, where $p_{1}^{(n-q)}(n)$ denotes the number of partitions of $n$ into exactly $n-q$ parts. In the first set there is only one term and its contribution to $p_{1}\left(n_{1}, \ldots, n_{k+1}\right)$ is equal to the coefficient of $x_{1}^{n_{1}} \ldots x_{k}^{n_{k}}$ in $c_{n_{k+1}} f_{1}\left(x_{1}, \ldots, x_{k}\right)$. Also, from (7) and Lemma 1, the 
coefficient of $x_{1}^{n_{1}} \ldots x_{k}^{n_{k}}$ in $c_{n_{k+1}} f_{1}\left(x_{1}, \ldots, x_{k}\right)$ is asymptotically equal to

$$
\begin{aligned}
\sum_{\left(n_{k+1}\right)} \prod_{m}\left(v_{m}^{\prime} !\right)^{-1} m^{-2 v_{m}^{\prime}}\left(\frac{6 n_{1}}{\pi^{2}}\right)^{\frac{1}{2}\left(R_{k}+\sum_{m}^{\prime} v_{m}^{\prime}\right)} \\
\times\left(4 \sqrt{3} n \prod_{l=2}^{k} n_{l} !\right)^{-1} \exp \left\{\pi /\left(\frac{2 n_{1}}{3}\right)\right\}\left\{1+\sum_{r=1}^{R_{k}+1+1} O\left(r n_{1}^{-\frac{1}{2}}\right)\right\} \\
=\left(\frac{6 n_{1}}{\pi^{2}}\right)^{\frac{1}{2} R_{k+1}}\left(4 \sqrt{ } 3 n_{1} \prod_{l=2}^{k+1} n_{l} !\right)^{-1} \exp \left\{\pi /\left(\frac{2 n_{1}}{3}\right)\right\}\left\{1+\sum_{r=1}^{R_{k+1}+1} O\left(r n_{1}^{-\frac{1}{2}}\right)\right\}
\end{aligned}
$$

provided that

$n_{k+1} ! \sum_{\left(n_{k+1}\right)} \prod_{m}\left(v_{m}^{\prime} !\right)^{-1} m^{-2 v_{m}^{\prime}}\left(\frac{6 n_{1}}{\pi^{2}}\right)^{\frac{1}{2}\left(\sum_{m}^{\prime} v_{m}^{\prime}-n_{k}+1\right)}=1+\sum_{r=1}^{R_{k+1}+1} O\left(r n_{1}^{-\frac{1}{2}}\right)$.

It is easily seen that any partition of $n_{k+1}$ into $n_{k+1}-q$ parts, where $q<\frac{1}{2} n_{k+1}$, must contain at least $n_{k+1}-2 q$ units. Therefore, for any particular partition $\sum_{m} m v_{m}^{\prime}$ of $n_{k+1}$ into $n_{k+1}-q$ parts, $\prod_{m} v_{m}^{\prime} ! \geqq \Lambda_{n_{k+1}-2 q}$, where $\Lambda_{n_{k+1}-2 q}=\left(n_{k+1}-2 q\right)$ ! for $q<\frac{1}{2} n_{k+1}$ and $\Lambda_{n_{k+1}-2 q}=1$ for $q \geqq \frac{1}{2} n_{k+1}$. Also, $p_{1}^{\left(n_{k+1}-q\right)}\left(n_{k+1}\right)=p_{1}(q)$ for $q \leqq \frac{1}{2} n_{k+1}$ and $p_{1}^{\left(n_{k+1}-q\right)}\left(n_{k+1}\right)<p_{1}(q)$ for $q>\frac{1}{2} n_{k+1}$. Hence, in order to prove (9), it is sufficient to show that

$$
\sum_{q=1}^{n_{k+1}-1} p_{1}(q)\left(\frac{6 n_{1}}{\pi^{2}}\right)^{-\frac{1}{2} q}\left(n_{k+1} !\right) \Lambda_{n_{k+1}-2 q}^{-1}=\sum_{r=1}^{R_{k+1}+1} O\left(r n_{1}^{-\frac{1}{2}}\right)
$$

The Hardy-Ramanujan formula (1) for $p_{1}(q)$ shows that, for all $q>0$,

$$
p_{1}(q)<C q^{-1} \exp \{\pi \sqrt{ }(2 q / 3)\} \text {. }
$$

Therefore,

$$
\begin{aligned}
\sum_{q=2}^{n_{k+1}-1} p_{1}(q) & \left(\frac{6 n_{1}}{\pi^{2}}\right)^{-\frac{1}{2} q}\left(n_{k+1} !\right) \Lambda_{n_{k+1}-2 q}^{-1} \\
& <C \sum_{q=2}^{n_{k+1}-1} q^{-1} \exp \left\{\pi /\left(\frac{2 q}{3}\right)\right\}\left(\frac{6 n_{1}}{\pi^{2}}\right)^{-\frac{1}{2} q} n_{k+1}^{2 q} \\
& =C \sum_{q=2}^{n_{k+1}-1} \exp \left\{\pi /\left(\frac{2 q}{3}\right)-\log q-\frac{1}{2} q \log \left(\frac{6 n_{1}}{\pi^{2} n_{k+1}^{4}}\right)\right\} \\
& <C \sum_{q=2}^{n_{k+1}-1} \exp \left\{-\frac{1}{4} q \log \left(\frac{6 n_{1}}{\pi^{2} n_{k+1}^{4}}\right)\right\} \\
& =O\left(n_{k+1}^{2} n_{1}^{-\frac{1}{2}}\right)
\end{aligned}
$$

since $n_{k+1}=o\left(n_{1}^{\frac{1}{2}}\right)$, and (10) follows immediately.

To complete the proof of (8), we have only to show that the contributions to $p_{1}\left(n_{1}, \ldots, n_{k+1}\right)$ from the other terms of $A_{n_{k+1}}$ can be neglected. By repeated applications of a similar argument to that employed in determining the coefficient of $x_{1}^{n_{1}} \ldots x_{k}^{n_{k}}$ in $c_{n_{k+1}} f_{1}\left(x_{1}, \ldots, x_{k}\right)$, we can show that the coefficient of 
$x_{1}^{n_{1}} \ldots x_{k}^{n_{k}}$ in $\prod_{m} c_{v_{m}} f_{1}\left(x_{1}, \ldots, x_{k}\right)$ is asymptotically equal to $\left(\frac{6 n_{1}}{\pi^{2}}\right)^{\frac{1}{2\left(R_{k}+\sum_{m} v_{m}\right)}}\left(4 \sqrt{ } 3 n_{1} \prod_{l=2}^{k} n_{l} ! \prod_{m} v_{m} !\right)^{-1}$

It therefore remains to show that

$$
\times \exp \left\{\pi /\left(\frac{2 n_{1}}{3}\right)\right\}\left\{1+\sum_{r=1}^{R_{k}+\sum v_{m}+1}{ }_{m}\left(r n_{1}^{-2}\right)\right\} .
$$

$$
n_{k+1} ! \sum_{\left(n_{k+1}\right)} \prod_{m}\left(v_{m} !\right)^{-1}\left(\frac{6 n_{1}}{\pi^{2}}\right)^{\frac{1}{2\left(2 v_{m}-n_{k+1}\right)}}=1+\sum_{r=1}^{R_{k+1}+1} O\left(r n_{1}^{-\frac{1}{2}}\right)
$$

and this follows in exactly the same manner as did (9). Finally, since $n_{l}=o\left(n_{1}^{\frac{1}{1}}\right)$ for $2 \leqq l \leqq j$, Theorem 1 is an immediate consequence of (8).

\section{Proof of Theorems 2, 3 and 4}

In (1), Hardy and Ramanujan obtained the asymptotic formula

$$
p_{3}\left(n_{1}\right)=\left(4.3^{t} n_{1}^{\frac{3}{4}}\right)^{-1} \exp \left\{\pi \sqrt{ }\left(n_{1} / 3\right)\right\}\left\{1+O\left(n_{1}^{-\frac{1}{2}}\right)\right\}
$$

as $n_{1} \rightarrow \infty$ and we can easily deduce, by a similar method to that employed by Nanda (5), that

$$
p_{3}\left(n_{1}, n_{2}\right)=\left(\frac{12 n_{1}}{\pi^{2}}\right)^{\frac{1}{n_{2}}}\left\{4 \cdot 3^{\frac{1}{4}} n_{1}^{\frac{1}{2}}\left(n_{2} !\right)\right\}^{-1} \exp \left\{\pi /\left(\frac{n_{1}}{3}\right)\right\}\left\{1+\sum_{r=1}^{n_{2}+1} O\left(r n_{1}^{-\frac{1}{2}}\right)\right\}
$$

as $n_{1} \rightarrow \infty$ for $n_{2}=o\left(n_{1}^{\frac{1}{1}}\right)$. The extension to the general $j$-partite number can be carried out exactly as in the proof of (8) and Theorem 3 follows immediately, since $n_{l}=o\left(n_{1}^{\frac{1}{1}}\right)$ for $2 \leqq l \leqq j$.

We now prove Theorems 2 and 4 . We denote by $p_{2}^{\left(n_{j}-q\right)}\left(n_{1}, \ldots, n_{j}\right)$ the number of different partitions of $\left(n_{1}, \ldots, n_{j}\right)$ into exactly $n_{j}-q$ parts in which no part has a zero component and we write $p_{4}^{\left(n_{j}-q\right)}\left(n_{1}, \ldots, n_{j}\right)$ for the number of partitions of $\left(n_{1}, \ldots, n_{j}\right)$ into exactly $n_{j}-q$ unequal parts in which no part has a zero component. For any particular partition $\sum_{m} m v_{m}$ of $n_{j}$ into exactly $n_{j}-q$ parts, the parts can be arranged in $\left(n_{j}-q\right) ! / \prod_{m} v_{m}$ ! distinguishable ways. If $\sum_{k=1}^{n_{j}-q} n_{l k}$ is any partition of $n_{l}$ into $n_{j}-q$ parts for $2 \leqq l \leqq j$, then the maximum number of distinct partitions of $\left(n_{1}, \ldots, n_{j}\right)$ into $n_{j}-q$ parts in the set

$$
\sum_{k=1}^{n_{j}-q}\left(n_{1 k}, m_{2 k}, \ldots, m_{j k}\right) \text {, }
$$

where, for $2 \leqq l \leqq j, m_{l 1}, \ldots, m_{l, n_{-}-q}$ run through the distinguishable arrangements of $n_{l 1}, \ldots, n_{l, n_{j}-q}$, is obtained when $\sum_{k=1}^{n_{j}-q} n_{1 k}$ is a partition of $n_{1}$ into unequal parts. It follows that

$$
p_{2}^{\left(n_{j}-q\right)}\left(n_{1}, \ldots, n_{j}\right) \leqq\left\{\left(n_{j}-q\right) !\right\}^{j-1} \prod_{l=1}^{j-1} p_{2}^{\left(n_{j}-q\right)}\left(n_{l}\right) \sum_{\left(n_{j}, q\right)} \prod_{m}\left(v_{m} !\right)^{-1},
$$


where the sum is taken over all partitions of $n_{j}$ into $n_{j}-q$ parts of the form $n_{j}=\sum_{m} m v_{m}$. We also have

$$
p_{4}^{\left(n_{j}-q\right)}\left(n_{1}, \ldots, n_{j}\right) \geqq\left\{\left(n_{j}-q\right) !\right\}^{j-1} \prod_{l=1}^{j-1} p_{4}^{\left(n_{j}-q\right)}\left(n_{l}\right) \sum_{\left(n_{j}, q\right)} \prod_{m}\left(v_{m} !\right)^{-1} .
$$

Since

$$
p_{4}^{\left(n_{j}-q\right)}\left(n_{1}, \ldots, n_{j}\right) \leqq p_{2}^{\left(n_{j}-q\right)}\left(n_{1}, \ldots, n_{j}\right)
$$

by definition, we obtain

$$
\begin{aligned}
& \left\{\left(n_{j}-q\right) !\right\}^{j-1} \prod_{l=1}^{j-1} p_{4}^{\left(n_{j}-q\right)}\left(n_{l}\right) \sum_{\left(n_{j}, q\right)} \prod_{m}\left(v_{m} !\right)^{-1} \leqq p_{4}^{\left(n_{j}-q\right)}\left(n_{1}, \ldots, n_{j}\right) \\
& \quad \leqq p_{2}^{\left(n_{j}-q\right)}\left(n_{1}, \ldots, n_{j}\right) \leqq\left\{\left(n_{j}-q\right) !\right\}^{j-1} \prod_{l=1}^{j-1} p_{2}^{\left(n_{j}-q\right)}\left(n_{l}\right) \sum_{\left(n_{j}, q\right)} \prod_{m}\left(v_{m} !\right)^{-1} .
\end{aligned}
$$

Next, we use the formula of Erdös and Lehner (2),

$$
p_{2}^{(k)}(n) \sim \frac{1}{k !}\left(\begin{array}{l}
n-1 \\
k-1
\end{array}\right)
$$

as $n \rightarrow \infty$ for $k=o\left(n^{\frac{1}{3}}\right)$, in the form, more convenient for our present purposes,

$$
p_{2}^{(k)}(n)=n^{k-1}\{k !(k-1) !\}^{-1}\{1+o(1)\} \text {. }
$$

We see that

$$
\begin{aligned}
p_{4}^{(k)}(n) & =p_{2}^{(k)}\left\{n-\frac{1}{2} k(k-1)\right\} \\
& =\left\{n-\frac{1}{2} k(k-1)\right\}^{k-1}\{k !(k-1) !\}^{-1}\{1+o(1)\} \\
& =n^{k-1}\{k !(k-1) !\}^{-1}\{1+o(1)\}
\end{aligned}
$$

as $n \rightarrow \infty$, provided that $k=o\left(n^{\frac{1}{3}}\right)$. Therefore, since $n_{j}=o\left(n_{l}^{\frac{1}{3}}\right)$ for $1 \leqq l \leqq j-1$, we obtain

$$
\begin{aligned}
& \left(n_{1} \ldots n_{j-1}\right)^{n_{j}-q-1}\left\{\left(n_{j}-q-1\right) !\right\}^{1-j} \sum_{\left(n_{j}, q\right)} \prod_{m}\left(v_{m} !\right)^{-1}\{1+o(1)\} \leqq p_{4}^{\left(n_{j}-q\right)}\left(n_{1}, \ldots, n_{j}\right) \\
& \leqq p_{2}^{\left(n_{j}-q\right)}\left(n_{1}, \ldots, n_{j}\right) \leqq\left(n_{1} \ldots n_{j-1}\right)^{n_{j}-q-1}\left\{\left(n_{j}-q-1\right) !\right\}^{1-j} \sum_{\left(n_{j}, q\right)} \prod_{m}\left(v_{m} !\right)^{-1}\{1+o(1)\}
\end{aligned}
$$

from (11). By putting $q=0$ in (12), we obtain

$$
\begin{aligned}
\left(n_{1} \ldots n_{j-1}\right)^{n_{j}-1} & \left\{\left(n_{j}-1\right) !\right\}^{1-j}\left(n_{j} !\right)^{-1}\{1+o(1)\} \leqq p_{4}^{\left(n_{j}\right)}\left(n_{1}, \ldots, n_{j}\right) \\
& \leqq p_{2}^{\left(n_{j}\right)}\left(n_{1}, \ldots, n_{j}\right) \leqq\left(n_{1} \ldots n_{j-1}\right)^{n_{j}-1}\left\{\left(n_{j}-1\right) !\right\}^{1-j}\left(n_{j} !\right)^{-1}\{1+o(1)\}
\end{aligned}
$$

and, since

$$
p_{2}\left(n_{1}, \ldots, n_{j}\right)=\sum_{q=0}^{n_{j}-1} p_{2}^{\left(n_{j}-q\right)}\left(n_{1}, \ldots, n_{j}\right)
$$

and

$$
p_{4}\left(n_{1}, \ldots, n_{j}\right)=\sum_{q=0}^{n_{j}-1} p_{4}^{\left(n_{j}-q\right)}\left(n_{1}, \ldots, n_{j}\right)
$$


we can see from (12) that Theorems 2 and 4 are proved if we show that $\sum_{q=1}^{n_{j}-1}\left(n_{1} \ldots n_{j-1}\right)^{-q}\left\{\left(n_{j}-1\right)\left(n_{j}-2\right) \ldots\left(n_{j}-q\right)\right\}^{j-1}\left(n_{j} !\right) \sum_{\left(n_{j}, q\right)} \prod_{m}\left(v_{m} !\right)^{-1}=o(1)$.

Now, since any partition of $n_{j}$ into $n_{j}-q$ parts, where $q<\frac{1}{2} n_{j}$, must contain at least $n_{j}-2 q$ units, we have $\prod_{m} v_{m} ! \geqq \Lambda_{n_{j}-2 q}$, where $\Lambda_{n_{j}-2 q}=\left(n_{j}-2 q\right)$ ! for $q<\frac{1}{2} n_{j}$ and $\Lambda_{n_{j}-2 q}=1$ for $q \geqq \frac{1}{2} n_{j}$. Also, $p_{2}^{\left(n_{j}-q\right)}\left(n_{j}\right)=p_{2}(q)$ for $q \leqq \frac{1}{2} n_{j}$ and $p_{2}^{\left(n_{j}-q\right)}\left(n_{j}\right)<p_{2}(q)$ for $q>\frac{1}{2} n_{j}$. Therefore, since the Hardy-Ramanujan formula (1) for $p_{2}(q)$ shows that, for all $q>0$,

$$
p_{2}(q)<C q^{-1} \exp \{\pi \sqrt{ }(2 q / 3)\}
$$

the left-hand side of (13) is less than

$$
\begin{aligned}
& C \sum_{q=1}^{n_{j}-1} q^{-1} \exp \left\{\pi \int\left(\frac{2 q}{3}\right)\right\}\left(n_{1} \ldots n_{j-1}\right)^{-q} n_{j}^{(j+1) q} \\
= & C \sum_{q=1}^{n_{j}-1} \exp \left\{\pi /\left(\frac{2 q}{3}\right)-\log q-q \log \left(\frac{n_{1} \ldots n_{j-1}}{n_{j}^{j+1}}\right)\right\} \\
< & C \sum_{q=1}^{n,-1} \exp \left\{-\frac{1}{2} q \log \left(\frac{n_{1} \ldots n_{j-1}}{n_{j}^{j+1}}\right)\right\}=o(1),
\end{aligned}
$$

since $n_{j}=o\left(n_{l}^{\frac{1}{3}}\right)$ for $1 \leqq l \leqq j-1$.

\section{REFERENCES}

(1) G. H. HaRdy and S. Ramanujan, Asymptotic formulæ in combinatory analysis, Proc. London Math. Soc. (2), 17 (1918), 75-115.

(2) P. ERDös and J. LEHNER, The distribution of the number of summands in partitions of a positive integer, Duke Math. J., 8 (1941), 335-345.

(3) F. C. Auluck, On partitions of bi-partite numbers, Proc. Cambridge Phil. Soc., 49 (1953), 72-83.

(4) G. MeInardus, Zur additiven Zahlentheorie in mehreren Dimensionen Teil I, Math. Annalen, 132 (1956), 333-346.

(5) V. S. Nanda, Bipartite partitions, Proc. Cambridge Phil. Soc., 53 (1957), 272-277.

(6) E. M. Wright, Partitions of multi-partite numbers, Proc. American Math. Soc., 7 (1956), 880-890.

(7) E. M. WRIGHT, The number of partitions of a large bi-partite number, Proc. London Math. Soc. (3), 7 (1957), 150-160.

(8) E. M. Wright, Partitions of large bipartites, American J. Math., 80 (1958), 643-658.

Department of Mathematics

UNIVERSITY OF ABERDEEN 